\title{
ANALYSIS OF THE OBSERVATIONAL CHARACTERISTICS OF SHADOW-EFFECTS IN THE JOVIAN DAM EMISSION
}

\author{
G. Litvinenko*, A. Konovalenko*, V. Zakharenko*, I. Vasylieva*, \\ P. Zarka ${ }^{\dagger}$, A. Lecacheux ${ }^{\dagger}$, V. Shaposhnikov ${ }^{\ddagger}$, H. O. Rucker ${ }^{\S}$, \\ M. Panchenko $\mathbb{I}$, and O. Ulyanov*
}

\begin{abstract}
Despite the fact that so-called "shadow effects" (bursts in absorption) in the Jovian Io-modulated decameter emission have been observed for the first time a long time ago, their physical origin still has not a clear understanding. This fact can be explained by the non-frequent appearance of such features in the Jovian DAM dynamic spectra. As result, analysis of the effects has minor interest and only a small number of researchers engaged a detailed study of them. In our opinion, the study of even such rare effects is important for understanding both the physical processes in the Jupiter radio emission sources and astrophysics in whole.

In the given work we present the analysis of the observational characteristics of shadow-effects in the Jovian DAM emission obtained by the UTR-2 radio telescope during the past and recent (January 2016) measurement campaigns. The dynamic range of the used receiving equipment was an order of magnitude higher than the observed signal level; that allows us to be sure that the obtained effects really belong to the Jovian DAM emission. The following parameters were studied: time-frequency scale for effect appearance, frequency bandwidth and time duration for simple event, variation of the burst width over the time axis, radiation intensity dependence in the time-frequency plane, sign and value of frequency drift, absorption depth (i.e. a selection of the galactic background level). We also made a simple comparative analysis of the specific properties of shadow effects in the Jupiter decameter emission with the parameters of the quasi-similar effect (absorption burst) of the solar radiation in decameter range.
\end{abstract}

\footnotetext{
* Institute of Radio Astronomy, National Academy of Sciences of the Ukraine

$\dagger$ LESIA, Observatoire de Paris, UMR CNRS, Meudon, France

$\ddagger$ National Research University High School of Economics, Nizhny Novgorod, Russia

$\S$ Commission for Astronomy, Austrian Academy of Sciences, Graz, Austria

I Space Research Institute, Austrian Academy of Sciences, Graz, Austria
} 
